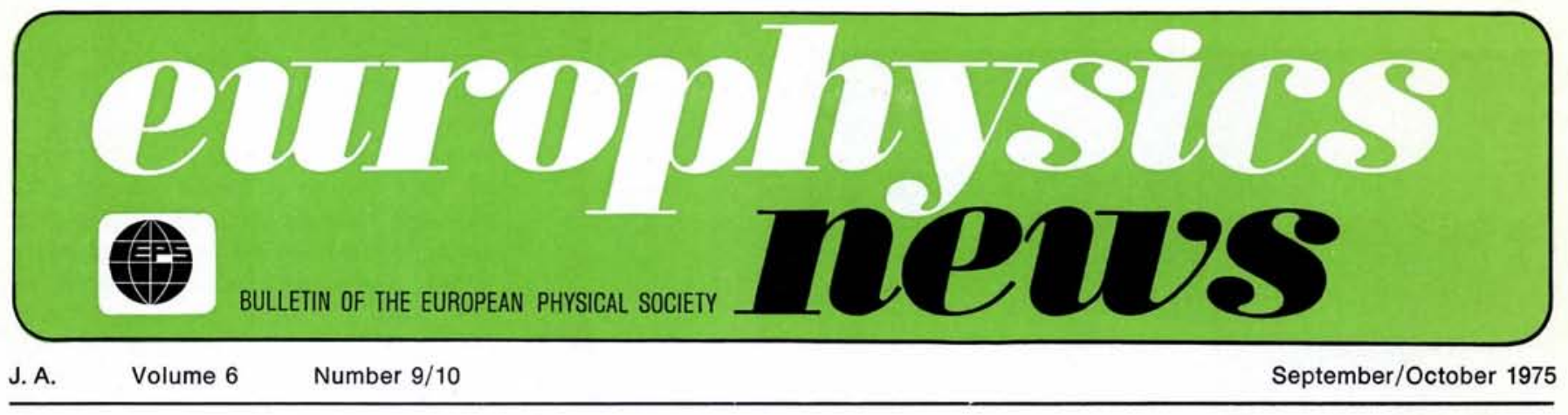

\title{
EPS Third General Conference
}

\author{
Bucharest, Romania, 9-12 September 1975
}

\begin{abstract}
"Science and technology are essential forces of progress... Physics must respond to the energy crisis, converting it from an apparent dead end into a turning point, leading to a new and steady equilibrium.» (I. Ursu)
\end{abstract}

\section{Energy and Physics}

In the splendid buildings of the campus of the Romanian National Centre of Physics outside Bucharest, the European Physical Society's third general conference on Energy and Physics took place between September 9 and 12 following meetings of the society's Executive Committee and the Council. In an ambience that was as efficient as it was agreeable, some 750 physicists from 27 countries participated, of whom about 300 came from West European countries, about 130 from the Eastern countries other than Romania, a dozen from outside Europe and the rest from the host state.

The significance of the conference was marked by the personal patronage of President Ceaucescu who held discussions with a number of the senior participants as well as sending to the Conference at its opening session a personal message through the Prime Minister. The proceedings were extensively covered in the Romanian press and there was no mistaking the real desire for effective cooperation within the physics community and for an increase of exchanges between the East and West. The will for collaboration is not confined to pure research but extends across the whole field to development and production. This was also evident from the exploratory meeting of
ACAPPI led by S. Kapitza who in the course of a review of $R \& D$ practices in the Soviet Union invited contacts with the Soviet State Committee on Science and Technology.

The efforts of the local committee under I. Ursu, epitomized by the untiring attention of I.A. Dorobantu, ensured that the work over so many months of the programme committee was fully exploited; it was notable that not only the plenary sessions in the morning were well attended but the parallel sessions and special meetings in the evening were equally full. In addition, when the formal proceedings ended for the day, there was no rush to leave the conference area and the corridors were animated with groups continuing the discussions.

\section{General consensus}

A criticism that could be made was that there was not enough time for discussion in the sessions and as a result, it might be rash to place too much emphasis on the apparent consensus of views. Nevertheless, the presentations had a sensitive coherency and an absence of partisanship which is unusual in such a burning subject as energy. It was also clear that there were no significant divisions on fundamentals between groups from idfferent nations or with different backgrounds.

It was not always the more aca-

\section{Contributors to this special} Bucharest Conference issue are:
B. Edmondson, Berkeley
W.T. Eeles, Capenhurst
O. Kofoed-Hansen, Geneva
W.S. Newman, Geneva
B. Robinson, Paris
E. Schatzman, Meudon
E.N. Shaw, Geneva
J.J. Went, Arnhem

In this summary of the Bucharest Conference, not all the subjects are reviewed, rather a number of authors have been asked to give their impressions of the highlights ; the editor will welcome comments from participants whose views differ from those presented here.

\section{CONTENTS}

EPS Third General Conference . 1 Use of Solar Energy _ . . . . 4 Transport and Storage of Energy 5 Nuclear Energy . . . . . . 7 Cosmological Energy . . . . 9 High Energy Physics . . . . 11 Energy Strategies . . . . . 12 International Conference on

Physics Education Reported at Bucharest . . . . . . 14

Society News . . . . . . . 15

Council Meeting in Bucharest . 16 


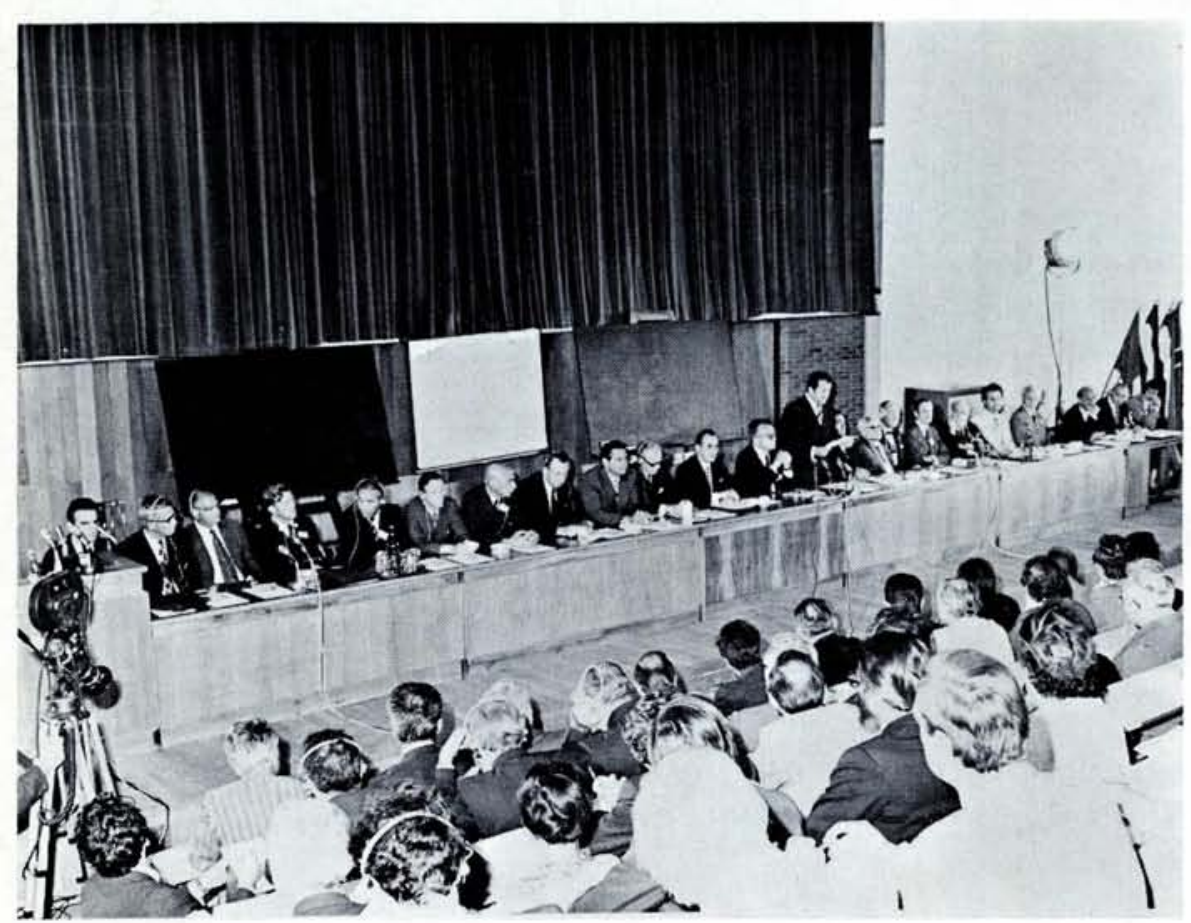

Opening session of EPS Third General Conference on Energy and Physics in Bucharest, Romania.

demic sessions which attracted the biggest audiences and many physicists took the opportunity of enlarging their appreciation of the overall energy problem. There was perhaps some surprise at the importance of some of the more mundane aspects of energy such as its transport and in particular the need for gas (notably hydrogen), or some of the more disagreeable consequences of economic considerations for, as N. Kurti emphasized, efficiency and economy were not the same. Equally some disappointment was apparent that there were no simple or ideal solutions immediately in view.

In this wide ranging conference, it was to be expected that many of the processes mentioned seemed to be on the fringe of, if not beyond, the realms of practicability. Some are feasible but limited in scale, others require such a tremendous degree of technical development that their practicability cannot yet be assessed. Curiously no place was given to a consideration of wave-power which is readily available off most of Western Europe at the requisite power densities and, seemingly, requires only development of existing technologies, rather than discovery of new ones.

\section{Basic considerations}

The scientific tone was quickly established by P.L. Kapitza in the opening session when he underlined the necessity for understanding the physical laws affecting energy conversion and transfer which had not been sufficiently taken into account in the past. A quite elementary study of the energy vector allowed one to draw certain conclusions of a quite explicit character. From simple reasoning, one could deduce the fact that because of the limited energy density, the direct conversion of chemical to electrical energy was not economic on a large scale; solar energy, whilst apparently unlimited and evidently interesting for special applications, was best exploited on a massive scale by passing through a carbon chain; geothermal applications were also limited because the heat density was so small; hydropower although ideal ecologically was only sensible if sufficient gravitational potential was available, otherwise it was better to use the area concerned for agriculture purposes. In reviewing the problems facing the exploitation of thermo-nuclear processes, he stated what might be considered one of the main messages of this conference, " it was better to understand the physics before building machines".

Not all the truths however are to be found in the laws of physics and the disparity in energy consumption between the more ideveloped and the less developed countries was one of the more unpalatable aspects of the energy situation. At the top end of the scale, energy consumption per person was $10 \mathrm{~kW}$, yet there were large areas where it was less than $0.1 \mathrm{~kW}$. Some $72 \%$ of the world's energy was consumed by less than $2 \%$ of the population and living standards seemed inextricably linked to the energy consumed. As physicists still believe in the laws of conservation, the word consumption is perhaps not the ideal scientific term but its meaning is clear. The disparity between nations was something that must concern us all and whilst there might seem to be a plethora of international organizations already concerning themselves with various aspects of the problem, S. Eklund would like to see an international energy institute created which would concern itself with this disparity and with problems on a global scale.

\section{Need for planning}

In planning ahead, it was necessary to draw up scenarios for future development but there was a need to clarify scenarios according to motivation and no single scenario could describe the coming situation; there must be continual updating as the situation changes. Another maxim from the conference was the statement that "it is dangerous to make predictions but one must nevertheless make plans" (C.M. Braams). In this respect, one could envy the position of the United States where ERDA haid begun to set out a clearly defined programme of research and development allocating $\$ 10 \mathrm{G}$ to a 5-year programme and establishing a new solar institute, whereas in Europe - at least in the West - a coherent energy development plan is conspicuous by its absence.

As a basis for calculation, W. Haefele proposed that one might consider the energy consumption as plateauing at a level of about $5 \mathrm{~kW} /$ person for a world population that had levelled off at about $13^{\prime} 000$ million in the year 2150 which would mean a global consumption of 65 TW. If there were a smooth growth from the present day, it would mean an integrated consumption of 100 TW year by 1990 , which would have grown to $3^{\prime} 000 \mathrm{TW}$ year in 2150 .

To meet this demand, it was going to be necessary to balance carefully what was available with what was acceptable. We shall need to burn a lot more coal but we were in danger of exceeding the tolerable carbon dioxide content of the troposphere, as recent studies have shown that $\mathrm{CO}_{2}$ accumulates far faster than was presupposed owing to the slow rate of exchange with the oceans. A study of the evolution of man's energy consumption in the past and the rate at which new sources could be introduced led one to the conclusion that nuclear energy had to start playing a major role now. But, no one 
strategy would be a panacea, many complementary solutions to the energy problem need to be found.

\section{Science only one aspect}

The sense of responsibility present at the conference was nowhere better expressed than in the Cecil Powell Memorial lecture given by Professor V.F. Weisskopf. He spoke of the frontiers and limits of science, explaining the one as being essentially a point of departure whilst the other was a constraint even though both were borders. During the past 500 years, science had been remarkably successful in concentrating its attention on limited studies which in due time led to the formulation of general laws. These detailed studies had allowed us to make a much deeper penetration in our uniderstanding of nature than the previous search for absolute truths.

There are two types of frontier which might be termed external and internal. The external frontier was the one beyond which we tried to go at a fundamental level, while the internal frontier covered the vast areas where the basic principles were known but the detail was not understood because of the inherent complexity. As one went up the scale from the elementary particles, either on the physical scale to the universe, or through the biological chain, another factor entered into it which he had dubbed funicity - the capacity for memory - where not only is the system complex but is dependent upon its history. He sees as one of the most important internal frontiers and the one most challenging to modern science the study of the brain.

But whilst it might be reasonable to assume that we shall be able to understand all the physical aspects of nature eventually, there will still be something left, we shall still not be describing our relations with it. Science can treat such things perhaps but not necessarily in a relevant way. Again, one might take as a message from the conference the phrase that "science sheds such a glaring light, it leaves shadows of ever deepening darkness". Science is only one side of our reactions to the universe. There are two pillars of our society: compassion and curiosity. Curiosity without compassion is inhuman and compassion without curiosity is ineffective.

But the translation of the compassion into practical terms is not easy as was evident at the evening discussion on the social aspects of scientific research. Although a big effort was made to keep the discussion to relatively narrow topics, and avoid darting off into polemics, it is a subject extremely hard to get to grips with and although the sincerity of the many speakers could not be questioned, the Advisory Committee on Physics and Society will have difficulty in extracting any clear policy for action.

\section{The jobs ahead}

It is perhaps better then, in this introduction to return to the easier subject of where the scientific priorities lie for physicists in these coming years affronting the problem of energy conversion, storage, distribution and conservation.

It was important to appreciate that resources were not the limit but it was a question of cost and the side effects in their exploitation. Taking as unit $Q=10^{21} \mathrm{~J}$ our present consumption was $Q / 4$ per year. In round terms coal resources amounted to $200 \mathrm{Q}$, geothermal $5 \times 10^{3} \mathrm{Q}$, nuclear energy (with the fast breeder) $5 \times 10^{6} \mathrm{Q}$, fusion (DT) $10^{7} Q$ and solar energy effectively unlimited. We were already concerned with the pollution caused by burning fossil fuels and the problems of nuclear energy had been given ample publicity, but all the new sources require conversion and all have high conversion losses with consequent ecological implications.

Haefele set out what he felt were the most important subjects for the immediate future and $\mathrm{A}$. Weinberg in reviewing the programme of the USA was able to confirm that this was generally in line with US thinking at the present time. He recalled Dixy Lee Ray's priorities aiming at energy independence, as conservation, synthetic production of oil and gas, further nuclear power and long range sources. Haefele put at the top of his list, by virtue of its importance, the problem of storage and this was followed by transport, the liquefaction of coal in situ (in order to produce transportable gas), the physics of the atmosphere and the dynamics of the ocean possibly leading to climatic control, the production of hydrogen including the study of electrolysis, means for capturing solar energy and then fusion.

Not all physicists may agree that these are the most significant tasks to be performed but there was no one in Bucharest disposed to argue and the President in his closing lecture chose to talk about physics - illuminated by his personal reminiscences rather than make didactic recommendations for the future.

E.N.S.
The Theory of Molecular Spectroscopy

Vol. I: The Quantum Mechanics and Group Theory of Vibrating and Rotating Molecules

by C. J. H. Schutte, Professor of Physical Chemistry, University of South Africa, Pretoria.

1975 about 568 pages

Price: US $\$ 70.95 /$ Dfl. 170.00

This book describes the mathematical theory of the spectroscopy of molecules and ions in the electronic ground state, in a way which is as self-contained as possible. The theory is presented in such a way that the book should appeal to the postgraduate student, as well as to the research worker in the fields of infra-red and Raman spectroscopy.

The first chapter surveys most of the mathematics needed in the later chapters on group theory. Special emphasis is laid on the theory of linear operators, their eigenvalue equations as well as their matrix representations.

The next chapter examines the mathematical theory of groups; here the accent is on the groups of operators which generate the molecular point groups that leave a point invariant. Complete discussions of the character tables, the correlation between a group and its suband super-groups, the point groups of non-rigid molecules, crystal groups and direct product groups are included.

Chapter 3 deals with the fundamentals of quantum mechanics particularly the harmonic/anharmonic oscillator, the quantisation of the orbital angular momentum.

After a full consideration of the classical Hamiltonian, Chapter 4 goes on to give a systematic presentation of the complete quantum mechanical molecular Hamiltonian operator for molecules. Next, those aspects of molecular quantum mechanics of importance to molecular interaction between dipole radiation and matter.

Volume II will deal with the spectroscopy of the various rotators, the vibrations of polyatomic molecules, particularly the use of projection operators and Green's theorem methods.

\section{NORTH-HOLLAND} PUBLISHING CO.

P.O. Box 211 Amsterdam, The Netherlands

Distributed in the U.S.A. and Canada by

American Elsevier Publishing Co. 52 Vanderbilt Ave, New York, N.Y. 10017 